\title{
Application of the boundary element method to 2D and 3D bubble dynamics
}

\author{
Z. Fu \& V. Popov \\ Wessex Institute of Technology, Environmental and Fluid Mechanics, \\ Southampton, UK
}

\begin{abstract}
The Boundary Element Method (BEM) was applied to the solution of bubble dynamics in an ideal fluid. Two-dimensional (2D) and three-dimensional (3D) models have been developed and the results have been compared to the analytical solution. The results show that only the 3D model can correctly represent the physics of the problem. The influence of the model parameters on the solution has been investigated for a single bubble.
\end{abstract}

Keywords: BEM, numerical simulation, bubble dynamics.

\section{Introduction}

A great amount of work has been conducted in the area of bubble dynamics which can be seen in the review papers by Plesset and Prosperetti [1] and Feng and Leal [2]. During the course of bubble oscillation, jet formation is a common phenomenon. As pointed out by Tsiglifis and Pelekasis [3], even when the bubble shape is initially very close to spherical, asymmetric collapse happens at the following stage and it gives rise to jet formation. The bubbles in the acoustic field can oscillate or they can collapse producing high temperatures and possibly jets. The creation of high temperature spots can help chemical reactions and this phenomenon is used in sonochemical reactors.

With advancement of the computational technology, numerical studies have been carried out in order to understand the bubble dynamics. One of the numerical techniques used is the Boundary Element Method (BEM) which possesses unique advantages in respect to other numerical techniques for these types of problems. Indirect BEM has been employed by Wang and Khoo [4] who pointed out that both source and dipole distribution methods encounter 
difficulties arising from hyper-singular integrals. By contrast, direct BEM solves the unknown (either potential or derivative) on the surface of the bubble in a direct way. One problem by applying the direct BEM is that the solution is only provided for the normal velocity on the bubble surface while the tangential components, which are also required, are not provided. Zhang et al. [5] applied a finite difference scheme to evaluate the tangential velocity (2D) or two tangential velocities (3D), thereby obtaining the material velocity on the bubble surface. This approach cannot guarantee sufficient accuracy for the velocity. When the simulation involves time history, errors accumulate and may result in computational instability.

In this work a BEM code has been developed to simulate bubble dynamics. The problem includes moving boundaries of the pulsating sphere which with time accumulates errors leading to numerical instabilities. A scheme has been applied in order to deal with the numerical instability and the model has been verified by comparison with the Rayleigh-Plesset equation.

\section{Description of the problem}

Let us consider a single bubble where the surrounding fluid represents the domain for numerical computation. The boundary of the problem is defined with the surface of the bubble, the fluid free surface and the solid structure(s) that may enclose the fluid or alternatively an imaginary boundary at infinity.

Bubble dynamics is essentially a time-dependent process, involving bubble expansion and collapse, bubble translation, jet formation, and possibly bubble separation and merging (for multiple bubbles). However, all of these mechanisms can be summarised as the consequence of the geometric evolution of the bubble surface, which is driven directly by the pressure difference between the bubble interior and exterior. In such sense, the problem is generally focused on the shape variation and moving of the bubble surface, which represents a moving boundary problem. The nature of the problem of bubble dynamics justifies the choice of the BEM numerical approach.

The boundary conditions involve generally the potentials and velocities. In case of the solid boundary, the normal velocities are set to be zero. The conditions on the bubble surface govern the bubble evolution. The subtle change of the boundary exerts highly sensitive outputs of velocities on the boundary; on the other hand, the normal velocities generated directly from BEM and the tangential velocities obtained from the potentials are used to update the geometry of the bubble surface. Hence both the geometry and the velocities on the boundary influence each other. As a result, the precision of boundary conditions determines how stable and accurate the numerical simulation is, as even small errors will be accumulated and will give rise to a bubble surface error as the time progresses.

The pressure governs the bubble surface evolution in time. Usually the pressure at the bubble interior can be described with a gas state equation. The pressure at the bubble exterior may be due to atmospheric pressure, hydraulic pressure, buoyancy forces, as well as the acoustic forcing. 


\section{Theoretical foundation}

\subsection{Boundary integral equation}

The fluid is assumed to be incompressible, homogeneous, inviscid and irrotational. The idealised fluid flow is governed by Laplace equation, i.e.:

$$
\nabla^{2} \phi=0 \text {, }
$$

where $\phi$ is the velocity potential of the fluid and the normal velocity on the surface of the bubble can be defined as $-\frac{\partial \phi}{\partial \vec{n}}$. Hence the above Laplace equation has the following representation in the integral form:

$$
c(\mathbf{p}) \phi(\mathbf{p})=\int_{\Omega}\left(G(\mathbf{p}, \mathbf{q}) \frac{\partial \phi(\mathbf{q})}{\partial \vec{n}}-\frac{\partial G(\mathbf{p}, \mathbf{q})}{\partial \vec{n}} \phi(\mathbf{q})\right) d \Gamma .
$$

The coefficient $\mathrm{c}(\mathbf{p})$ is a function of the solid angle of the boundary at the collocation point $\mathbf{p}$ (see Figure 1). With $\mathbf{p}$ located inside the domain, $\mathrm{c}(\mathbf{p})$ is fixed as 1.0 and for $\mathbf{p}$ on a smooth boundary $\mathrm{c}(\mathbf{p})$ becomes $1 / 2$. However, the boundary is more realistically of irregular shape, which requires repeated laborious computation for $\mathbf{c}(\mathbf{p})$. Alternatively, $\mathrm{c}$ is calculated in an indirect way by physically considering a constant potential, which is explained in more detail in the book by Brebbia and Dominguez [6]. G stands for the fundamental solution:

$$
G(\mathbf{p}, \mathbf{q})=\left\{\begin{array}{cc}
-\frac{1}{2 \pi} \operatorname{In}(|\mathbf{p}-\mathbf{q}|) & \text { for } 2 \mathrm{D} \\
\frac{1}{4 \pi|\mathbf{p}-\mathbf{q}|} & \text { for } 3 \mathrm{D}
\end{array}\right.
$$

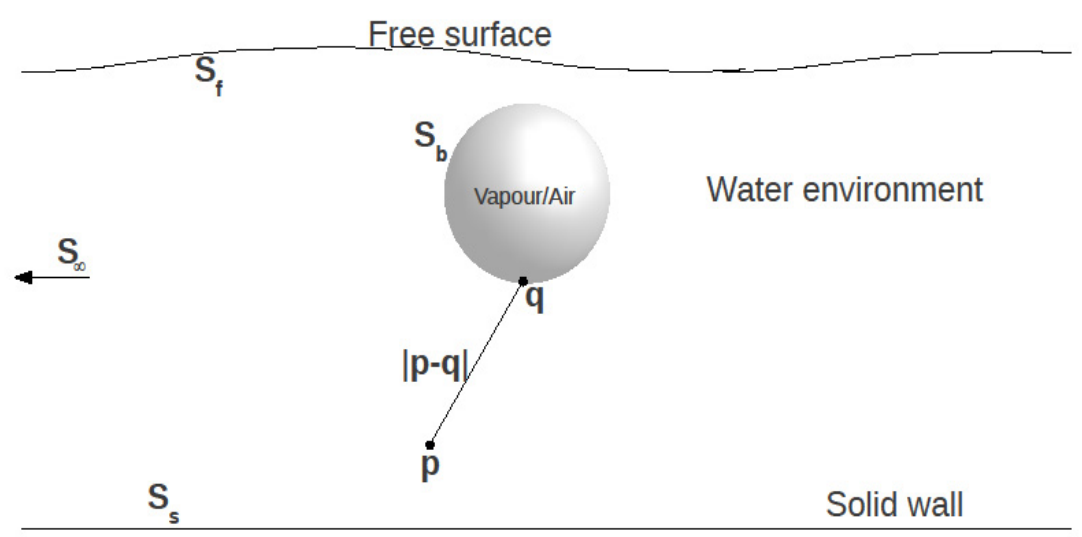

Figure 1: The general structure for modelling with boundary integral equation where the boundary is made up of the bubble surface $\mathrm{S}_{\mathrm{b}}$, the free surface $\mathrm{S}_{\mathrm{f}}$, the solid boundary $\mathrm{Ss}$ and the imaginary boundary at infinity $S_{\infty}$. 


\subsection{Bernoulli equation}

The boundary integral equation is capable of solving the bubble problems in a stationary state. When it comes to bubble oscillation with time, the modified Bernoulli equation should be introduced to describe the dynamic boundary conditions at the interface. By neglecting the effects of viscosity, buoyancy force and surface tension, the Bernoulli equation is expressed as follows:

$$
\frac{D \widetilde{\phi}}{D \widetilde{t}}=-\frac{1}{2}|\nabla \widetilde{\phi}|^{2}+\frac{1}{\widetilde{\rho}_{l}} \Delta \widetilde{p},
$$

where $\Delta \tilde{p}$ is the pressure difference on the boundary. Note that the symbol ' $\sim$ ' represents the physical quantity of a parameter; without ' $\sim$ ' it indicates a dimensionless quantity. For any point $\mathbf{q \in S}$ (see Figure 1), the particle velocity is:

$$
\widetilde{\mathbf{u}}=\frac{D \widetilde{\mathbf{q}}}{D \widetilde{t}}=-\nabla \widetilde{\phi} .
$$

Meanwhile, the pressure difference at the bubble interface follows:

$$
\Delta \widetilde{p}=\widetilde{p}_{b}-\widetilde{p}_{a}-\widetilde{p}_{\infty}
$$

where $\tilde{p}_{\boldsymbol{b}}, \tilde{p}_{\boldsymbol{a}}$ and $\tilde{p}_{\infty}$ are the pressure at the bubble interior, the standard atmospheric pressure and the pressure at infinity.

We consider a bubble that embraces air (assumed to be ideal gas) and vapour. The bubble interior is assumed uniform in terms of pressure, temperature and composition. In addition, the idealised gas has a polytrophic behaviour. Then the internal pressure follows the gas state equation:

$$
\widetilde{p}_{b}=\widetilde{p}_{v}+\widetilde{p}_{0}\left(\frac{\widetilde{V}_{0}}{\widetilde{V}}\right)^{\gamma},
$$

where $\tilde{p}_{v}$ is the vapour pressure assumed constant, $\tilde{p}_{0}$ is the initial pressure contributed by air with the corresponding initial bubble volume $\tilde{V}_{0}$, and $\gamma$ is the ratio of specific heat (see Figure 2). The standard atmosphere pressure $\tilde{p}_{\boldsymbol{a}}$ is applied as the pressure scale. In addition, we prescribe the length and time scales as $\tilde{V}_{0}^{\frac{1}{3}}$ and $\tilde{V}_{0}^{\frac{1}{3}} \sqrt{\tilde{\rho}_{l} / \tilde{p}_{a}}$, respectively, then Bernoulli equation is obtained in the following form:

$$
\frac{D \phi}{D t}=-\frac{1}{2}|\nabla \phi|^{2}+p_{0}\left(\frac{1}{V}\right)^{\gamma}-1-p_{\infty} .
$$



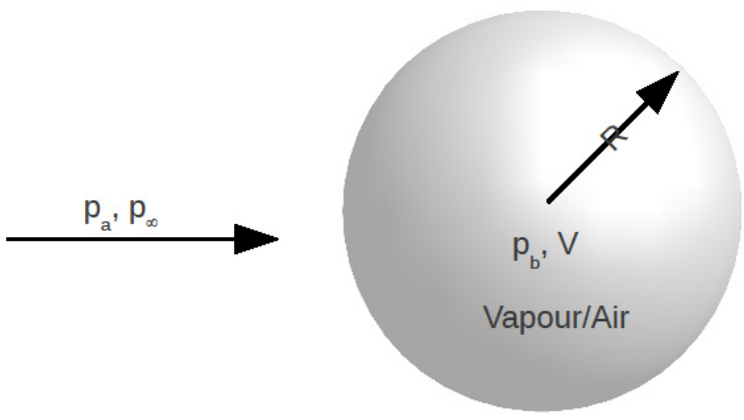

Figure 2: A spherical bubble composed of vapour and air with the pressure, temperature and composition evenly distributed.

\section{Numerical scheme}

\subsection{Numerical procedure}

The initial state of the bubble(s) should be prescribed to carry out the numerical simulation. In case of a single spherical bubble, Blake and Gibson [7] proposed a mathematical formula to obtain a reasonable initial potential on the boundary. This formula can be derived from the Rayleigh-Plesset equation:

$$
\widetilde{\phi}_{0}=\widetilde{R}_{0}\left\{\frac{2}{3}\left(\frac{\widetilde{p}_{\infty}-\tilde{p}_{b}}{\widetilde{\rho}_{l}}\right)\left[\left(\frac{\widetilde{R}_{m}}{\widetilde{R}_{0}}\right)^{3}-1\right]\right\}^{\frac{1}{2}},
$$

where $\tilde{R}_{m}$ is the maximum radius that the bubble can reach under the constant pressure difference $\Delta \tilde{p}=\tilde{p}_{b}-\tilde{p}_{\infty}$. The above equation sets both the initial radius and the maximum radius as known and it is beneficial as a predictable maximum bubble size can be used as an important parameter for reference during the bubble oscillation. Nonetheless, the formula is only restricted to the vapour bubble with constant internal pressure which is driven by a constant pressure forcing from outside. More generally, it is more reasonable to prescribe the initial conditions of the bubble(s) as stationary, i.e. $\mathbf{u}=-\nabla \phi=0$, or written in another way, the normal potential derivatives $\frac{\partial \phi}{\partial \vec{n}}$ on the boundary are known. Hence the corresponding potentials can be obtained by discretising the boundary and constructing the matrices so as to solve the integral equations with Neumann boundary conditions. Thereafter, the Runge-Kutta method is employed to update the potentials on the boundary. Once new potentials on the boundary are determined, the normal potential derivatives can be calculated. Both the potentials and their derivatives are used to obtain the material velocities on the bubble surface and the geometry of the bubble surface is ready for update. On the same principle to update both the potentials and the bubble geometry, repetition is carried out likewise and the bubble oscillation with a clear time history is depicted. However, special care should be taken during 
implementation of the numerical techniques in order to guarantee the accuracy of results and the stability of the model.

The developed programme allows us to select different orders and types of elements. Herein, the quadratic quadrilateral element is put forward for illustration. When the boundary is discretised, the information is created on the number of nodes and elements, together with the arrangement of nodes in each individual element. The employed order of nodes in an element can be seen in Figure 3, which determines not only the order of interpolation coefficients $\Phi_{i}$, but also the direction of the unit normal. The interpolation coefficients are given as:

$$
\begin{gathered}
\Phi_{1}=\frac{1}{4} \eta_{1}\left(\eta_{1}-1\right) \eta_{2}\left(\eta_{2}-1\right), \Phi_{2}=\frac{1}{2}\left(1-\eta_{1}{ }^{2}\right) \eta_{2}\left(\eta_{2}-1\right) \\
\Phi_{3}=\frac{1}{4} \eta_{1}\left(1+\eta_{1}\right) \eta_{2}\left(\eta_{2}-1\right), \Phi_{4}=\frac{1}{2} \eta_{1}\left(1+\eta_{1}\right)\left(1-\eta_{2}{ }^{2}\right), \\
\Phi_{5}=\frac{1}{4} \eta_{1}\left(1+\eta_{1}\right) \eta_{2}\left(1+\eta_{2}\right), \Phi_{6}=\frac{1}{2}\left(1-\eta_{1}{ }^{2}\right) \eta_{2}\left(1+\eta_{2}\right) \\
\Phi_{7}=\frac{1}{4} \eta_{1}\left(\eta_{1}-1\right) \eta_{2}\left(1+\eta_{2}\right), \Phi_{8}=\frac{1}{2} \eta_{1}\left(\eta_{1}-1\right)\left(1-\eta_{2}{ }^{2}\right) \\
\Phi_{9}=\left(1-\eta_{1}{ }^{2}\right)\left(1-\eta_{2}{ }^{2}\right)
\end{gathered}
$$

where both $\eta_{1}$ and $\eta_{2}$ are in the range of $(-1,1)$. Meanwhile, the right-hand rule is applied for the direction of the unit normal, as shown in Figure 3. The unit normal vector with respect to the surface is given as:

$$
\frac{\partial \mathbf{q}\left(\eta_{1}, \eta_{2}\right)}{\partial \vec{n}}=\left(\sum_{i=1}^{9} \frac{\partial \Phi_{i}}{\partial \eta_{1}} \cdot \mathbf{q}_{i}\right) \times\left(\sum_{i=1}^{9} \frac{\partial \Phi_{i}}{\partial \eta_{2}} \cdot \mathbf{q}_{i}\right) \text {. }
$$
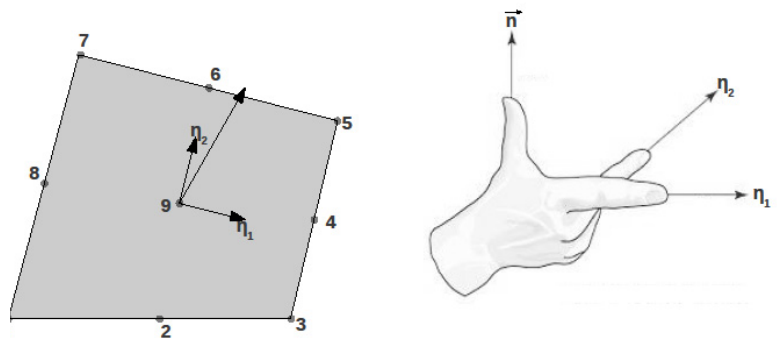

Figure 3: The position of nodes on an element and the normal vector determined with the right-hand rule.

After dicretisation of the boundary, the system matrix coefficients can be evaluated and the matrix can be assembled. With the application of quadratic quadrilateral elements, the potential and the derivatives ought to be interpolated from (10). Correspondingly, (2) has the following discretised form: 


$$
\begin{aligned}
c(\mathbf{p}) \phi(\mathbf{p})= & \sum_{i=1}^{m}\left\{\int_{s_{i}}\left\{G(\mathbf{p}, \mathbf{q}) \sum_{j=1}^{9}\left[\Phi_{j} \frac{\partial \phi_{j}}{\partial \vec{n}}\right]\right\} d \Gamma\right\} . \\
& -\sum_{i=1}^{m}\left\{\int_{s_{i}}\left\{\frac{G(\mathbf{p}, \mathbf{q})}{\partial \vec{n}} \sum_{j=1}^{9}\left[\Phi_{j} \phi_{j}\right]\right\} d \Gamma\right\} .
\end{aligned}
$$

where $\mathrm{m}$ stands for the number of elements on the boundary. Integration on each element $S_{i}$ is operated by isoparametric Gaussian quadrature. When the collocation point is allocated on the integrated element as a singularity point, an extra measure should be taken. Chahine and Perdue [8] evaluated the strongly singular integrals analytically. In this work, the third-degree coordinate transformation proposed by Telles [9] has been employed.

Once the unknown field variables are obtained from (12), the evaluation for the next time step can begin. The time scheme is constructed by the Bernoulli equation with the form of ordinary differential equations. To secure high accuracy, a fifth-order Runge-Kutta formula is employed. The step size is adaptive rather than constant, which depends on the embedded criterion (see Cash and Karp [10]). The scheme is given in (14):

$$
\begin{gathered}
k_{1}=h f\left(x_{n}, y_{n}\right) \\
k_{2}=h f\left(x_{n}+a_{2} h, y_{n}+b_{21} k_{1}\right) \\
\cdots \\
k_{6}=h f\left(x_{n}+a_{6} h, y_{n}+b_{61} k_{1}+\cdots+b_{65} k_{5}\right) \\
y_{n+1}=y_{n}+c_{1} k_{1}+c_{2} k_{2}+c_{3} k_{3}+c_{4} k_{4}+c_{5} k_{5}+c_{6} k_{6}+O\left(h^{6}\right)
\end{gathered}
$$

where the coefficients $\mathrm{a}, \mathrm{b}$ and $\mathrm{c}$ are constant and known, for more details see Press et al. [11]. However, (8) tells us that $\frac{D \phi}{D t}$ cannot be updated between the current and next time steps, as both the velocity and pressures have yet to be determined. Hence, one more physical criterion is established as follows:

$$
\Delta t_{\text {max }}=\frac{d \phi}{\max \left\{\left.\left|-\frac{1}{2}\right| \nabla \phi\right|^{2}+p_{0}\left(\frac{1}{V}\right)^{\gamma}-1-p_{\infty} \mid\right\}},
$$

where the denominator on the right hand side of the equation above is the maximum value of $\frac{D \phi}{D t}$ along the whole boundary. With the predefined maximum potential jump d $\phi$, the $\Delta t$ is available as the maximum step size to the next time step. Generally $\mathrm{d} \phi$ is set at the level of $\mathrm{O}\left(10^{-2}\right)$, as shown previously by Taib [12] who used 0.08 and by Wang et al. [13] who used 0.03 .

\subsection{The smoothing scheme}

The implementation of surface smoothing is one of the key points to maintain the stability during the simulation. We introduce a spherical single bubble. When it is subjected to symmetric pressure in a infinitely open field, the spherical shape is preserved. Nonetheless, the inevitable tiny errors for each time step accumulate and the bubble gradually loses its spherical shape. In case of 2D, the 
curve becomes saw-teeth shaped, whist convex and concave appear alternately on the surface of the $3 \mathrm{D}$ bubble (see Figure 4).

The implemented smoothing scheme is based on the technique of least squares. The basic idea is to predefine the smoothing function in advance with a certain number of unknown coefficients. Then a greater number of nodes are picked up around the targeted nodes to be smoothed. Once the least squares problem is solved, the new coordinate is determined by the smoothing function.

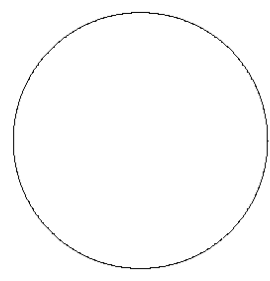

(a)

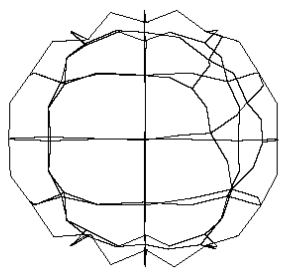

(c)

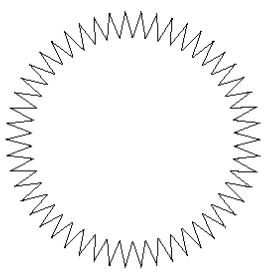

(b)

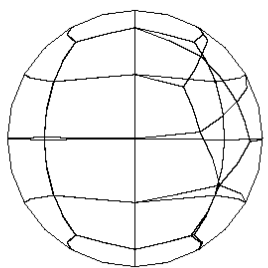

(d)

Figure 4: The meshes for a single bubble in two dimension and three dimension: (a) 2D before smoothing; (b) 2D after smoothing; (c) $3 \mathrm{D}$ before smoothing; (d) 3D after smoothing.

For $2 \mathrm{D}$, the function is in the form of a polynomial representation referred to a local coordinate system:

$$
y_{l}^{\prime}=a_{0}+a_{1} x_{l}+a_{2} x_{l}^{2},
$$

while the function for $3 \mathrm{D}$ is represented by a biquadratic equation as:

$$
z_{l}^{\prime}=a_{0}+a_{1} x_{l}+a_{2} y_{l}+a_{3} x_{l}^{2}+a_{4} x_{l} y_{l}+a_{5} y_{l}^{2},
$$

where the symbol "' indicates values after smoothing. In the case of a $2 \mathrm{D}$ problem, three unknown coefficients are included in the function; hence we pick up four neighbouring nodes in addition to the targeted node itself. By contrast, the way to select nodes in the $3 \mathrm{D}$ case is a bit more complex. More than six nodes ought to be selected in accordance with the unknowns in the smoothing function. We employed the approach proposed by Zhang et al. [5]. Firstly, we find 1 nodes which are immediately neighbouring to the targeted nodes. Then with reference to each of the 1 nodes, the neighbouring nodes in the second level are found, adding up m nodes. Finally, the third level of neighbouring nodes are found in the same way based on the m nodes at the second level, in this case we 
denote them with $\mathrm{n}$. Therefore, we have in total $1+\mathrm{m}+\mathrm{n}+1$ nodes to smooth the boundary involving targeted nodes.

To guarantee the serviceability of smoothing, the principle should be strictly followed that the new normal vector at the targeted point should be the same or highly similar to the old one before smoothing. Zhang et al. [5] suggested an iterative method to compute the new normal vector, based on which the local frame is determined.

Moreover, the potential is also required for smoothing to avoid a 'jump' from one node to its neighbouring nodes for which the same scheme applies. Figure 4 displays comparison of the bubble before and after smoothing.

\section{Verification and analyses}

\subsection{Verification of the model}

The developed model is capable of simulating bubble oscillation in both two and three dimensions. Before carrying out the investigation on the bubble dynamics, the model should be verified. Herein the Rayleigh-Plesset equation is introduced as an analytical solution for the bubble oscillation which is compared to the one obtained by using the model.

Further we refer to the Rayleigh-Plesset equation as the 'Rayleigh-Plesset bubble'. The Rayleigh-Plesset bubble is assumed to be perfectly spherical with a radius $R_{0}$ at the initial state $t=0$. The Rayleigh-Plesset equation is represented by:

$$
R \ddot{R}+\frac{3}{2} \dot{R}^{2}=p_{b}-1-p_{\infty} .
$$

Substituting $\mathrm{p}_{\mathrm{b}}$ with the dimensionless form of (7), the equation above is modified as:

$$
R \ddot{R}+\frac{3}{2} \dot{R}^{2}=p_{v}+p_{0}\left(\frac{R_{0}}{R}\right)^{3 \gamma}-1-p_{\infty} .
$$

To compare the results, input values are used as shown in Table 1. The radius variation with time is shown in Figure 5. The results show that the bubble oscillation based on the 3D model is in agreement with that of the RayleighPlesset bubble. However, the bubble from the 2D model oscillates with apparently different frequencies and phases, despite of the similar amplitude. Therefore, the 3D model proves its applicability for modelling the bubble dynamics, whilst the 2D model apparently cannot be used, as it represents oscillation of an infinite cylinder rather than a 3D sphere.

Table 1: $\quad$ Input values for verification of bubble oscillation.

\begin{tabular}{ll|ll}
\hline $\mathrm{R}_{0}$ & 0.1 & $\gamma$ & 1.4 \\
\hline $\mathrm{p}_{\mathrm{v}}$ & 1.0 & $\mathrm{p}_{0}$ & 2.0 \\
\hline $\mathrm{p}_{\infty}$ & 2.0 & & \\
\hline
\end{tabular}




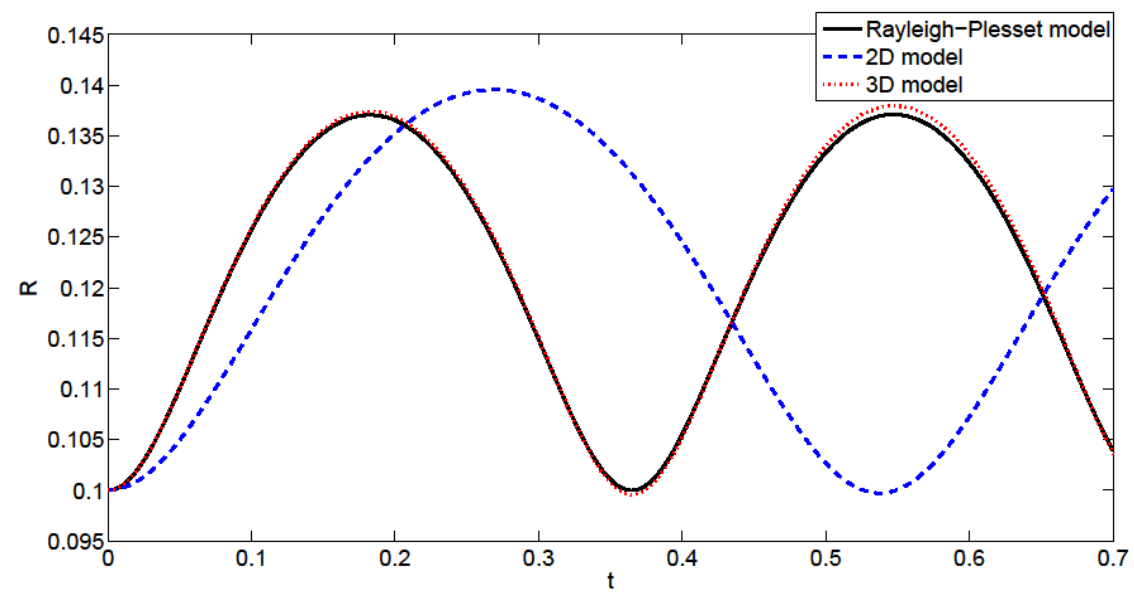

Figure 5: The radius variation of the oscillating bubble against time where three curves correspond to results from the Rayleigh-Plesset equation, 2D model and 3D model.

\subsection{Oscillation of a single bubble}

This example investigates the oscillation of a single bubble located in an infinite domain. The following factors are investigated: the initial bubble size, the air pressure at the bubble interior and the initial bubble-fluid interface velocity (i.e. $\nabla \phi)$.

Figure 6 depicts bubble oscillations of different initial radii. The amplitude tends to be bigger when the initial radius of the bubble increases. Also, the bigger initial bubble possesses a lower frequency. Meanwhile the oscillating frequency decreases when the pressure amplitude increases (Figure 7).

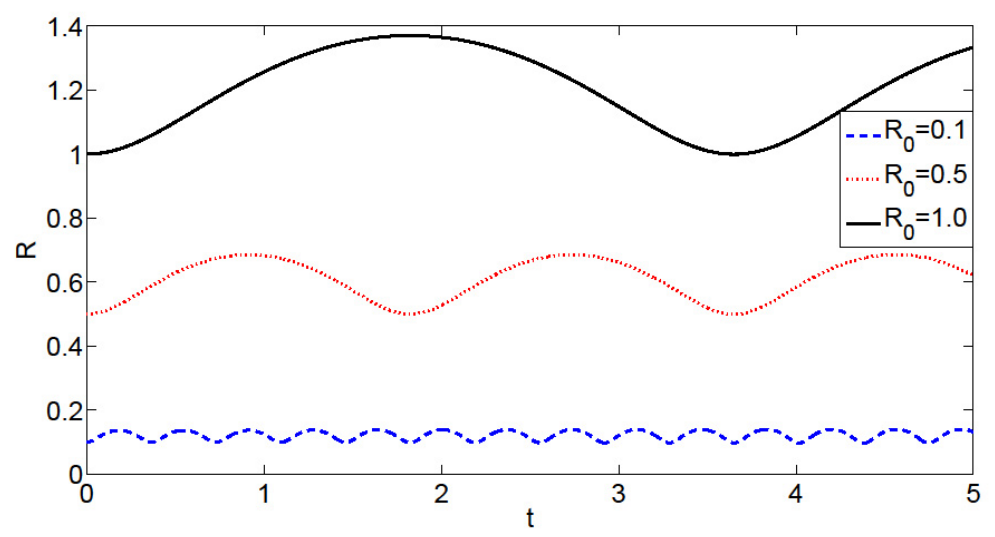

Figure 6: The oscillation of single bubbles with different initial radii $\left(\mathrm{p}_{0}=2.0\right.$ and $\nabla \phi=0)$. 


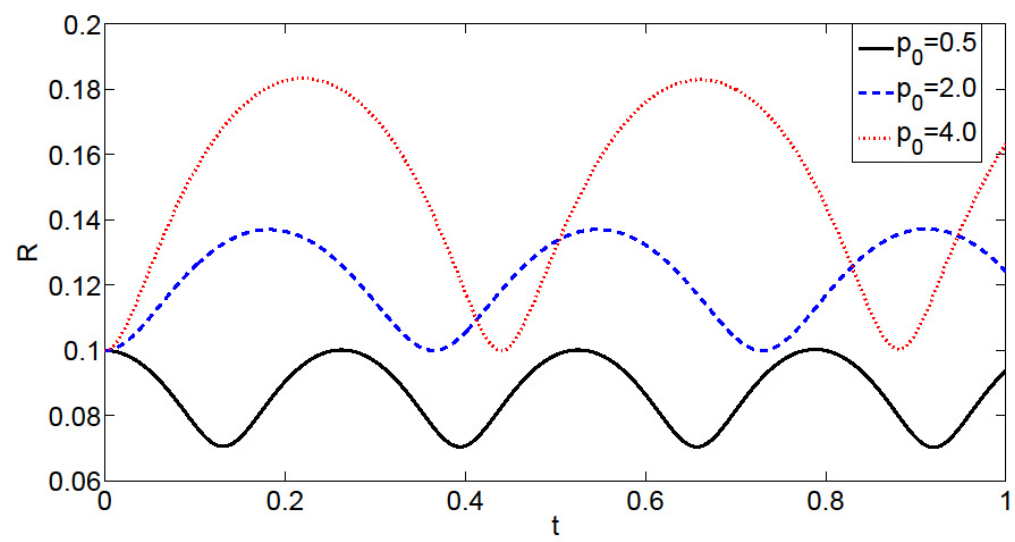

Figure 7: The oscillation of single bubbles with different initial air pressures $\left(\mathrm{R}_{0}=0.1\right.$ and $\left.\nabla \phi=0\right)$.

The influence of the initial bubble-fluid interface velocity on the bubble dynamics may be comparatively more complicated. Even the same amplitude of the velocity may not guarantee an identical process. As shown in Figure 8, plot (a) is for initial normal velocity forcing the bubble to expand, and the higher normal velocity produces higher oscillation amplitude. When the interface velocity direction is set towards the bubble interior, the bubble initially compresses corresponding to the velocity direction and then it expands. Both plots show that the initial velocity of the interface enlarges the amplitude of the oscillations.

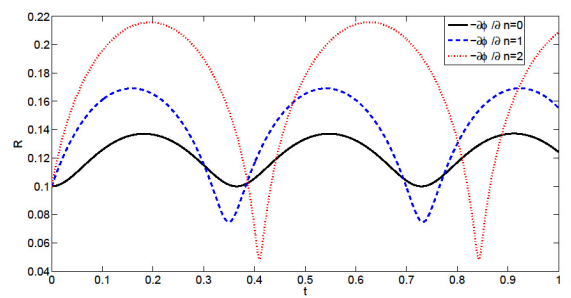

(a)

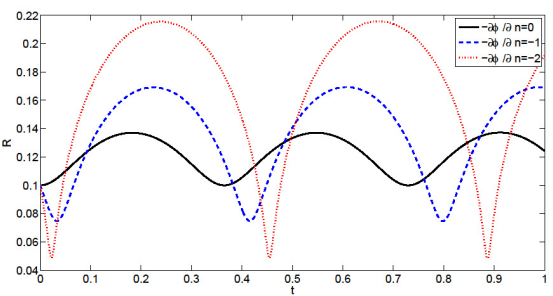

(b)

Figure 8: The oscillation of a single bubble with different initial interface velocities $\left(\mathrm{p}_{0}=2.0\right.$ and $\left.\mathrm{R}_{0}=0.1\right)$. (a) Velocity towards exterior; (b) velocity towards interior.

\section{Conclusions}

A numerical model has been developed for the problem of bubble dynamics. The model has been developed for the $2 \mathrm{D}$ and $3 \mathrm{D}$ cases. The model has been verified by using the Reyleigh-Plesset equation for oscillation of single bubble. The comparison of the results of the $2 \mathrm{D}$ and $3 \mathrm{D}$ models with the Reyleigh-Plesset equation shows that the $2 \mathrm{D}$ model cannot be used to accurately predict the 
behaviour of the bubbles, while the $3 \mathrm{D}$ model is in good agreement with the analytical solution. Once verified the model has been used to investigate the influence of several parameters on the single bubble dynamics.

\section{Acknowledgement}

The present study was supported by the SONO project, contract number: 228730, as part of the Seventh Framework Programme (FP7-NMP-2008-Large2).

\section{References}

[1] Plesset, M.S. \& Prosperetti, A., Bubble dynamics and cavitation. Ann. Rev. Fluid Mech., 9, pp. 145-185, 1977.

[2] Feng, Z.C. \& Leal, L.G., Nonlinear bubble dynamics. Annu. Rev. Fluid Mech., 29, pp. 201-243, 1997.

[3] Tsiglifis, K. \& Pelekasis, N.A., Numerical simulations of the aspherical collapse of laser and acoustically generated bubbles. Ultrasonics Sonochemistry, 14, pp. 456-469, 2007.

[4] Wang, C. \& Khoo, B.C., An indirect boundary element method for threedimensional explosion bubbles. Journal of Computational Physics, 194, pp. 451-480, 2004.

[5] Zhang, Y.L., Yeo, K.S., Khoo, B.C. \& Wang, C., 3d jest impact and toroidal bubbles. Journal of Computational Physics, 166, pp. 336-360, 2001.

[6] Brebbia, C.A. \& Dominguez, J., Boundary elements an introductory course. Computational Mechanics Publications, Southampton, UK, 1989.

[7] Blake, J.R. \& Gibson, D.C., Growth and collapse of a vapour cavity near free surface. J. Fluid Mech., 111, pp. 124-140, 1981.

[8] Chahine, G.L. \& Perdue, T.O., Simulation of the three-dimensional behaviour of an unsteady large bubble near a structure. In Proc. Third Int. Colloquium on bubbles and drops, Monterey, California, 1988.

[9] Telles, J.C.F., A self-adaptive co-ordinate transformation for efficient numerical evaluation of general boundary element integrals. International journal for numerical methods in engineering, 24, pp. 959-973, 1987.

[10] Cash, J.R. \& Karp, A.H., A variable order Runge-Kutta method for initial value problems with rapidly varying right-hand sides. ACM Transactions on Mathematical Software, 16, pp. 201-222, 1990.

[11] Press, W.H., Teukolsky, S.A., Vetterling, W.T. \& Flannery, B.P. Numerical recipes in Fortran 77. Cambridge University Press, 2nd edition edition, 2001.

[12] Taib, B.B., Boundary integral method applied to cavitation boundary dynamics. PhD thesis, The University of Wollongong, Wollongong, New South Wales, USA, 1985.

[13] Wang, Q.X., Yeo, K.S., Khoo, B.C. \& Lam, K.Y., Nonlinear interaction between gas bubble and free surface. Comput. Fluids, 25, pp. 607-628, 1996. 